\title{
Modified Circular Layer Caching in Wireless Sensor Networks
}

\author{
Sajpreet Kaur ${ }^{1}$ and Sukhwinder Singh ${ }^{2}$ \\ GNDU, RC, Jalandhar ${ }^{1}$ \\ UIET, PU, Chandigarh ${ }^{2}$ \\ Sajpreet.dhillon@gmail.com,sukhdalip@pu.ac.in
}

\begin{abstract}
The cutback in the energy loss during communicating the sensed data in WSN is needed to support the long term sensor applications. The caching of the data at intermediate nodes offers a good approach to improve the energy efficiency of WSN. In this paper, the Modified Concentric Circular Layer caching scheme is proposed which maintains a sink table to store the Id's of the nodes caching the sensed data. Thus the cached data is retrieved from caching nodes by searching the sink table, leading to reduction in number of queries injected into network to discover the cached data. The proper cache management scheme is used that ensures cache coherence and consistency. The different factors affecting any caching scheme are also analyzed. The simulation results show energy savings ranging from 23\% - 82\% under different scenarios over the already existing scheme.
\end{abstract}

Keywords: Caching, Energy Efficiency, Zigbee, WSN

\section{Introduction}

With the advent of MEMS technology, it has become possible to design small size sensor nodes that can be easily deployed under different conditions to monitor the environment [1]. The sensor nodes deployed in an area to sense and report the changes collectively forms a Wireless Sensor Networks (WSN). The Energy consumption in communicating the sensed data to the sink is the major concern of these networks. Much of the work is done in improving the energy efficiency of WSN. Many routing protocols [2], compression [3] and aggregation techniques [4] have been designed to handle the energy constraints. Caching can also be used as one of the approach that can yield fruitful results to achieve the energy efficiency in WSN.

Caching aims at storing the sensed data in the intermediate nodes between the sink and source so that every time instead of asking from the source, the data can be retrieved from the nodes caching the data, this reduces the communication overhead and hence saves energy.

Various caching schemes have been proposed to take advantage of caching the sensed data to provide efficient WSN. These schemes vary in the selection of nodes to cache the sensed data. N. Chand et al. proposes a novel cluster cooperative (CC) scheme in which the information of all the nodes lying within a cluster is handled by the "super" node called cache state node (CSN) [5]. D. Nikos et al. presents NiCoCa protocol in which the nodes forming the central part of the network are given the role of mediator to coordinate the caching decisions [6] whereas in T.P. Sharma et al. proposed DRDD cooperative caching that utilizes dual radio based antennas to cache data at the nodes along the data/query path, using virtual grid construction [7]. J. Xu et al. proposes EEWC which gives a waiting cache which increases energy efficiency by cutting down traffic flow by dividing the network into clusters [8]. Dae-Young Kim et al. proposes an active caching 
technique that aims at providing the desired communication reliability $(\mathrm{CR})$ during the multihop communication over the sensor networks [9]. K. Almi'ani et al. makes use of Mobile Element (ME) to find the subset of nodes that act acts as caching points [10]. N. Chauhan et al. proposes scheme called Global Cluster Cooperation strategy in Mobile Adhoc Networks in which information regarding each and every node is maintained at cluster level [11]. N. Hoeller et al. proposes Dynamic Adaptive Caching Scheme (DACS) to handle energy efficiency by caching data [12].

Caching of the data in the nodes near the sink or forming the central part of network shows better approach of caching the data $[6,7,14]$.

This paper discusses modified Concentric Circular Layer Caching scheme which uses cooperative caching strategy to cache the data in the nodes surrounding the sink along with the table maintained at each sink. The cache admission, cache discovery and cache invalidation scheme is also discussed that offers indispensible contribution to the success of any caching scheme.

Rest of the paper is organized as follows. Section 2 discusses the system environment. Section 3 discusses the query processing of the requested data. The result and analysis along with the simulation parameters is discussed in the Section 4. The entire work is concluded in Section 5.

\section{System Environment}

\subsection{Network Model}

The entire network consists of large number of homogeneous sensor nodes capable of sensing and communicating with each other with their radio signals. The entire sensor field is assumed as two-dimensional plane where each node is aware of its own location and coordinates (x,y) which serve as a node identification number [13]. The sensor field is divided into grid of equal square sized cells where radio range is used to decide the size of the cell.

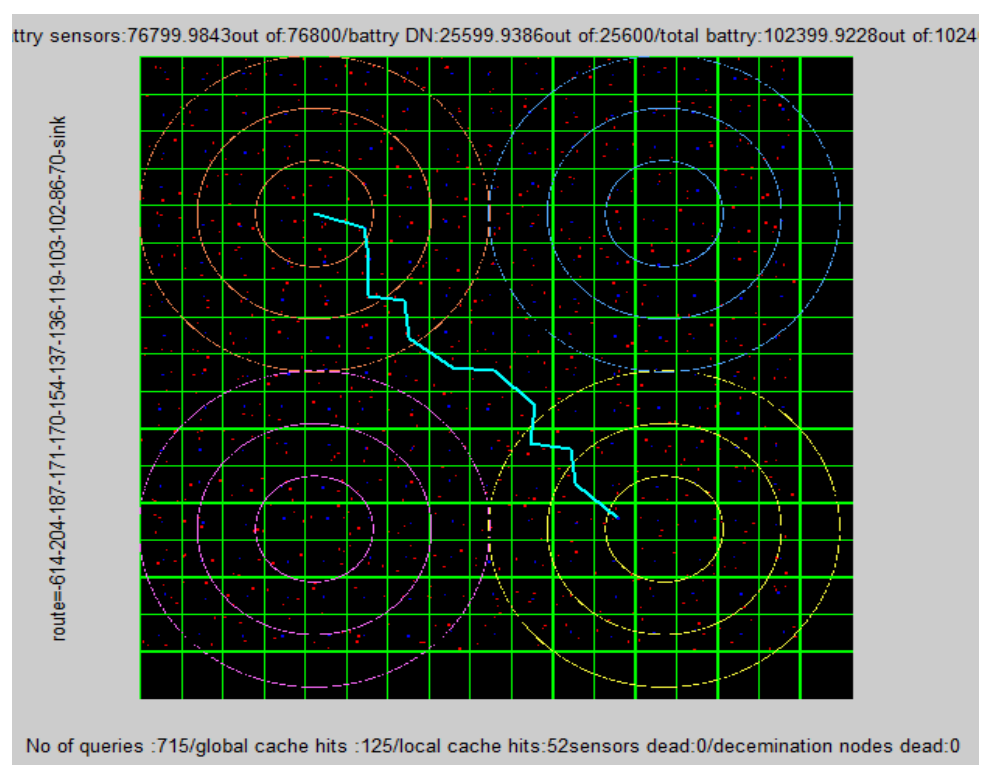

Figure 1. Network Setup

Each cell of a grid acts as a cluster and the node lying close to the centre of the cell is assigned the task of cluster head. The cluster head is also known as dissemination node (DN) which collects the data from the sensing sources of its cluster and forwards the data 
to the sink. Each DN selects the next DN according to the routing algorithm employed to forward data. Thus the DN collectively forwards the data from the sources to the sink.

Figure 1 depicts the designed network scenario in which the green coloured lines represent the grid dividing the entire simulation area into small cells. The red and blue coloured spheres in each cell represents sensing sources and cluster heads respectively. The size of each cell is determined by the radio range supported by the radio model of the sensor node. The cyan coloured line represents the routing of data from the source to the sink which is done based on routing algorithm.

The Concentric Circle represents the implemented caching scheme to improve the energy efficiency of the sensor networks.

\subsection{Modified Concentric Circular Layer Caching Scheme}

S. Pant et al. proposes a caching scheme in which the Concentric Circular layers (CCL) are formed around each sink and the various nodes lying in the layers formed acts as a cumulative cache to store the sensed data [14]. The algorithm used to form the CCLs around the sink, floods a message to all nodes lying at a particular distance from the sink, which forms a cache layer by responding to the message to cache the sensed data.

In the proposed modified caching scheme, sink table is maintained at sink that keeps track of all the cached data items along with their respective caching nodes. The concentric layers are formed in accordance to the communication ranges as supported by the used ZigBee Pro S2 series [16].

2.2.1. Cache Admission Policy: This policy defines which data item to cache and the technique used to cache the data items in the caching node. In the proposed caching scheme, Token based Admission policy is used in which the node holding the token will cache the data. The token is passed from innermost layer to the outermost layer. The layer holding the token is known as Active Cache layer (ACL). Once the last layer is reached, the token is passed back to the innermost layer and the nodes having free cache are searched for caching the data. If there are no free nodes to further cache the data, then a cache invalidation policy is used to replace the cached data items.

2.2.2. Cache Discovery Policy: Cache discovery is used to efficiently discover and deliver the requested data item from the neighboring nodes to the sink. In the proposed scheme, Table based Cache Discovery policy is used. The table maintained at sink is searched to find out the queried data item. The structure of sink table is shown below:-

Table 1. Structure of Sink Table

\begin{tabular}{|c|c|c|c|c|}
\hline $\begin{array}{c}\text { Circular } \\
\text { Layer Id } \\
(2 \text { bits })\end{array}$ & $\begin{array}{c}\text { Caching } \\
\text { Node Id } \\
(16 \text { bits })\end{array}$ & $\begin{array}{c}\text { Source Node } \\
\text { Id } \\
(16 \text { bits })\end{array}$ & $\begin{array}{c}\text { TTL } \\
(4 \text { bits })\end{array}$ & $\begin{array}{c}\text { Time of data } \\
\text { acquisition } \\
(4 \text { bits })\end{array}$ \\
\hline
\end{tabular}

Whenever any data item is cached, its entry is maintained at the sink table. Each entry consists of Circular Layer Id field which contains the layer number to which caching node belongs, Caching Node Id is the id of the node caching the queried source data, Source Node Id is the id of the node whose data is being cached, TTL field contains the time duration during which the collected data will be fresh and can be considered as valid data and Time of data acquisition field contains the time value at which the source data was collected. Cache Consistency is maintained by removing the cached data item entry from the sink table and corresponding caching node when it's associated TTL value expires. This helps in maintaining the latest and valid value of data item in the caching nodes. 
In the proposed scheme, Direct Cache Retrieval scheme is proposed to retrieve the cached data item from the caching nodes. Whenever any sink injects the query for particular source data, the sink table is searched by Source Node ID to find whether the requested data item is cached or not. If an entry is found, cache hit is said to occur and corresponding Caching Node Id is referred by sink to directly retrieve the data from the caching node by setting its communication range in accordance to the layer to which the caching node belongs.

Caching node stores much information along with the data values. The structure of the caching node is depicted in Table 2.

Table 2. Structure of Caching Node

\begin{tabular}{|c|c|c|c|c|c|}
\hline $\begin{array}{c}\text { Source ID } \\
(16 \text { bits })\end{array}$ & $\begin{array}{c}\text { Data } \\
\text { Value } \\
(8 \text { bits })\end{array}$ & $\begin{array}{c}\text { Data } \\
\text { Value } \\
(8 \text { bits })\end{array}$ & $\begin{array}{c}\text { Data Value } \\
(8 \text { bits })\end{array}$ & $\begin{array}{c}\text { Time of data } \\
\text { acquisition } \\
(4 \text { bits })\end{array}$ & $\begin{array}{c}\text { TTL } \\
(4 \text { bits })\end{array}$ \\
\hline
\end{tabular}

The data value is the value of the sensed data. The data value may correspond to any parameter depending upon the application area for which sensor nodes have been deployed. Time of data acquisition and TTL is used to invalidate the cache entry when there exists no free cache to store the new sensed data. This scheme exploits the sink table to increase the energy efficiency of the caching scheme by replacing the query flooding approach of cache discovery scheme with the Table based Cache Discovery scheme.

2.2.3. Cache Invalidation Policy: This policy is used to invalidate the cached items when there is no free space available to store new data. Not all the nodes in cache layer will have free space. Rather than waiting for the total number of nodes in cache layer to become free, the emphasis is given on the amount of available free cache in each layer. But sometimes a point comes when all the caching nodes of all layers are full. Then the cache invalidation scheme starts invalidating the cached data from the innermost layer. The cache invalidation is done on the basis of TTL. TTL associated with every data item defines its validity period. The data entry that was cached earlier will be replaced first. Apart from this, a trigger based concept is used in which whenever sink invalidates any entry in its table, at the same time that entry will be invalidated from the caching node too.

\section{Query Processing}

The proposed caching scheme is implemented in the multiple sink scenarios. The network is simulated such that any sink can randomly query any source by injecting a query into network. First the querying sink will check its own sink table to find the requested data, if it is found then a local cache hit is said to occur. If cache miss occurs, then the actual sink of the queried source is located to get the desired data item. If the data is retrieved from the actual sink cache, then global cache hit is said to occur. If the data is not supplied by any caching node then it will be retrieved from the source by using routing algorithm and will be cached in the caching nodes of both the querying and actual sink for future use. Further request for the same data will be replied back by the caching node.

\section{Results and Discussions}

The entire scheme is simulated in MATLAB 2011a. The energy efficiency of the proposed modified scheme is compared with the CCL [14] scheme under the simulated environment. 


\subsection{Simulation Parameters}

The simulation parameters considered for evaluating the proposed work are presented in Table 3 and Table 4.

\section{Table 3. Simulation parameters of} Proposed Network

\section{Table 4. Energy Requirements of the sensor node for communicating data}

\begin{tabular}{|l|c|}
\hline \multicolumn{1}{|c|}{ Parameters } & Values \\
\hline Sensor Field Region & $3600 * 3600$ \\
\hline Number Of Nodes & 1024 \\
\hline Number Of Sinks & 4 \\
\hline Query Packet Size & 30 Bytes \\
\hline Data Packet Size & 30 Bytes \\
\hline Flash Memory & 128 Bytes \\
\hline Simulation Time & $500 \mathrm{sec}$ \\
\hline
\end{tabular}

\begin{tabular}{|c|c|c|c|}
\hline $\begin{array}{c}\text { Power } \\
\text { Level }\end{array}$ & $\begin{array}{c}\text { Range } \\
(\mathrm{m})\end{array}$ & $\begin{array}{c}\text { Transmitting } \\
\text { Power }(\mu \mathrm{J})\end{array}$ & $\begin{array}{c}\text { Receiving Power } \\
(\mu \mathrm{J})\end{array}$ \\
\hline 1 & 300 & 6.14 & 1.13 \\
\hline 2 & 600 & 9.60 & 1.76 \\
\hline 3 & 900 & 15.36 & 2.82 \\
\hline
\end{tabular}

The simulation parameters are taken in accordance with the designed network scenario and ZigBee ProS2 series standard.

The Energy requirements are calculated using ZigBee specifications and ZigBee datasheet [15-16]. The entire calculation of the energy consumption for the proposed scheme is carried out using the above specified energy requirements of the sensor node.

\subsection{Result and Analysis}

The performance of any caching scheme is affected by many factors. Caching of data is done in those applications in which frequency of change in sensed data value is very low. So that data can be made available by caching node when needed in future by the other sink. The following factors are analyzed that affect any caching scheme and total cache hits:

1. Time-To-live (TTL):- defines the expiry time of the sensed data value. The source sensing the data associates this value with each sensed data as per the expected frequency of change in value of that data. If the value of TTL is low, then the cached data will become obsolete soon and hence caching of that data will be useless.

2. Number of Queries injected: - Caching is greatly affected by the number of queries injected into network. If the rate of number of queries being injected into network is less, then the chances of querying the same source is greatly reduced. Hence the cached data will never be used and the basic purpose of caching the sensed data is violated.

3. Number of Sources: - The number of sources being queried for collecting the sensed data also determines the cache hit ratio. When all the sources are queried then the chances of querying the same source are bleak and hence the cache hit ratio gets affected. When the number of sources for collecting the data is reduced then the chances of querying the same source increases manifold and leads to increase in cache hit ratio.

Caching scheme when analyzed in light of these factors show considerable variation in results taken on the basis of evaluation parameters.

The detailed analysis of energy consumption of the proposed scheme and CCL scheme [14] under different scenarios along with the energy savings attained by our proposed scheme is presented in the Table 5 . 
Table 5. Energy Consumption of the Modified Caching Scheme and CCL [14] Scheme under Different Scenarios

\begin{tabular}{|c|c|c|c|c|}
\hline $\begin{array}{c}\text { Sources } \\
(\%)\end{array}$ & $\begin{array}{c}\text { TTL } \\
(\%)\end{array}$ & $\begin{array}{c}\text { Energy } \\
\text { Consumption } \\
\text { in Modified } \\
\text { Caching (\%) }\end{array}$ & $\begin{array}{c}\text { Energy } \\
\text { Consumption } \\
\text { in CCL [14] } \\
(\%)\end{array}$ & $\begin{array}{c}\text { Energy } \\
\text { Savings (\%) }\end{array}$ \\
\hline 100 & 25 & 38.42 & 61.57 & 23.15 \\
\hline 100 & 50 & 33.72 & 66.28 & 32.56 \\
\hline 100 & 65 & 29.49 & 70.51 & 41.02 \\
\hline 75 & 25 & 29.64 & 70.36 & 40.72 \\
\hline 75 & 50 & 21.97 & 78.03 & 56.06 \\
\hline 75 & 65 & 18.61 & 81.39 & 62.78 \\
\hline 50 & 25 & 25.64 & 74.36 & 48.73 \\
\hline 50 & 50 & 17.87 & 82.13 & 64.26 \\
\hline 50 & 65 & 15.67 & 84.32 & 68.65 \\
\hline 25 & 25 & 18.38 & 81.62 & 63.25 \\
\hline 25 & 50 & 11.51 & 88.49 & 76.98 \\
\hline 25 & 65 & 8.53 & 91.48 & 82.95 \\
\hline
\end{tabular}

The amount of savings in energy consumption that can be achieved is application dependent for which caching scheme is used. The applications having TTL associated with data more than $50 \%$ and number of sources less than $75 \%$ saves considerable amount of energy with the modified caching approach over the CCL [14] scheme. By considering TTL value as $50 \%$ and number of sources as $75 \%$ as the best scenario, the energy savings of $56.05 \%$ has been achieved by the modified caching scheme. Thus considering it as the best scenario, the proposed scheme offered almost half time more energy savings which would greatly enhance the energy efficiency of any sensor network applications.

\section{Conclusion}

In this paper the modified Circular layer Caching scheme is discussed. The proposed scheme shows improved energy efficiency by reducing the amount of queries injected into network while discovering the cached data items. The presence of requested data value in the cache is checked by searching the sink table instead of flooding the query request to all the caching nodes to retrieve it. The sink directly retrieves requested data from the caching node by establishing direct communication with the caching node. The various factors affecting the total number of cache hits in any caching scheme are also analyzed. The proposed scheme can be further enhanced by implementing mobility of the nodes in the network scenario.

\section{References}

[1] I. F. Akyildiz, W. Su, Y. Sankarasubramaniam and E. Cayirci, "Wireless sensor networks: a survey", IEEE Communications Magazine, vol. 38, no. 4, (2002) March 15, pp. 393-422.

[2] K. Akkaya and M. Younis, "A survey on routing protocols for wireless sensor networks", Elsevier Ad Hoc Networks, no. 3, (2005), pp. 325-349.

[3] N. Kimura and S. Latifi, "A Survey on Data Compression in Wireless Sensor Networks", International Conference on Information Technology: Coding and Computing, vol. 2, (2005) April, pp. 8-13.

[4] E. Fasolo, M. Rossi, J. Widmer and M. Zorzi, "In-network aggregation techniques for wireless sensor networks: a survey”, IEEE Wireless Communications, vol. 14, no. 2, (2007) April, pp. 70-87.

[5] N. Chand, R. C. Joshi and M. Misra, "An Efficient Caching Strategy in Mobile Ad Hoc Networks Based on Clusters", IEEE, (2006). 
[6] D. Nikos, K. Daimitrios and M. Yannis, "Cooperative Caching in Wireless Multimedia Sensor Networks", Third International Mobile Multimedia Communication Conference (Mobimedia’07), (2007) August.

[7] T. P. Sharma, R. C. Joshi and M. Misra, "Dual Radio based Cooperative Caching for Wireless Sensor Networks", $16^{\text {th }}$ IEEE Conference, (2008), pp. 1-7.

[8] J. Xu, K. Li, Y. Shen and J. Liu, "An Energy-Efficient Waiting Caching Algorithm in Wireless Sensor Network", International Conference on Embedded and Ubiquitous Computing, vol. 1, (2008), pp. $323-$ 329.

[9] D.-Y. Kim and J. Cho, "Active Caching: A Transmission Method to Guarantee Desired Communication Reliability in Wireless Sensor Networks", IEEE communications letters, vol. 13, no. 6, (2009) June.

[10] K. Almi'ani, J. Taheri and A. Viglas, "Data Caching Approach for Sensor Applications", IEEE International Conference on Parallel Distributed and Computing", Applications and Technologies, (2009), pp. 88-93.

[11] N. Chauhan, L. K. Awasthi, N. Chand, R. C. Joshi and M. Misra, "Global Cluster cooperation strategy in Mobile Adhoc Networks", International Journal of Computer Science and Engineering, vol. 2, no. 7, (2010).

[12] N. Hoeller, C. Reinke, J. Neumann, S. Groppe, F. Frischat and V. Linnemann, "DACS: A Dynamic Approximation Caching Scheme", IEEE 5th International Conference on Digital Information Management (ICDIM 2010), (2010) July, pp. 339-346.

[13] Y. Xu, J. Heidemann and D. Estrin, "Geography-informed Energy Conservation for Ad Hoc Routing", International Conference on Mobile Computing and Networking, (2001), pp. 70-84.

[14] S. Pant, N. Chauhan, N. Chand, L. K. Awasthi, and B. B. Dubey, "Improving Fading-Aware Routing with Circular Cache Layers in Wireless Sensor Networks", Springer-Verlag Berlin Heidelberg, (2011), pp. 395-402.

[15] M. Simek and P. Moravek, "Modeling of Energy Consumption of Zigbee Devices in Matlab Tool", Elektrorevue Journal, vol. 2, no. 3, (2011), September.

[16] http://www.digi.com.

\section{Authors}

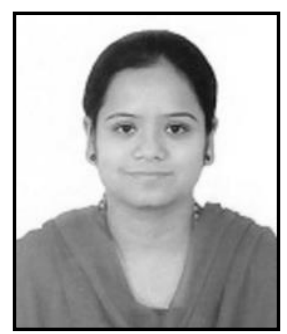

Sajpreet Kaur, she has done M.E. in Computer Science \& Engineering from UIET, Panjab University, Chandigarh. Currently, she is working as Assistant Professor at Guru Nanak Dev University, Regional Campus, Jalandhar. Her areas of interest include Wireless Sensor Networks, Network Security, and Energy Conservation in WSN.

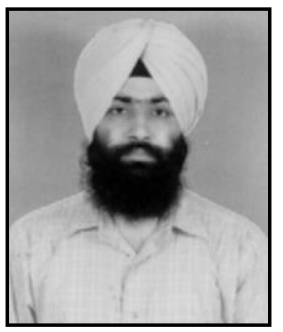

Sukhwinder Singh, he received his Ph.D. degree in Computer Science Engineering from IIT Roorkee. He is Professor in Department of computer science and engineering at University Institute of Engineering and Technology, Panjab University Chandigarh, India. He has published more than 20 research papers in reputed journals and conferences. His areas of Interest are Image compression, Biomedical Image processing, Network security and wireless sensor networks. 
International Journal of Sensor and Its Applications for Control Systems Vol.3, No.1 (2015) 